demonstrated that calprotectin, a heterodimer of S100A8 and S100A9, expressed and secreted during monocyte infiltration into inflamed tissues, is a good biomarker of treatment responses in AS. Here, we aimed to determine if calprotectin levels are predictive for radiographic spinal progression in AS patients.

Materials and Methods 76 AS patients were selected from German Spondyloarthritis Inception Cohort (GESPIC). Spinal radiographs were scored by two readers using the modified Stoke Ankylosing Spondylitis Spine Score (mSASSS) system. Subsequently, anteroposterior views of the lumbar spine were scored for presence of syndesmophytes. Radiographic spinal progression was defined as 1) mSASSS worsening by $\geq 2$ units after 2 years, and 2) development of a new syndesmophyte or progression of existing syndesmophytes after 2 years. Serum calprotectin levels were determined by ELISA.

Results High calprotectin levels were associated with mSASSS worsening over two years in AS, with an Area Under the Curve (AUC) of 0.740 (95\% CI $0.614-0.866 ; P=0.004)$. The odds ratio (OR) for radiographic spinal progression (mSASSS worsening by $\geq 2$ units) in patients with calprotectin serum level $>0.5 \mu \mathrm{g} / \mathrm{ml}$ was 6.2 (95\% CI 1.6-24.2, $P=0.009$ ). The association between calprotectin levels and mSASSS progression remained significant after adjusting for other independent risk factors (syndesmophytes at baseline, elevated C-reactive protein (CRP), and smoking): $\mathrm{OR}=5.5$ (95\% CI $1.2-25.8 ; P=0.030)$. Analysis of syndesmophyte formation and/or progression as outcome for structural damage yielded similar results: calprotectin levels were significantly associated with progression of syndesmophytes. The AUC was 0.670 (95\% CI $0.520-0.819 ; P=0.031)$. The predictive value of calprotectin was independent but similar to that of CRP.

Conclusions Calprotectin is an independent predictor for radiographic spinal progression in AS.

\section{A4.12 ENDOTHELIN-1 PROMOTES THE ENDOTHELIAL-TO-MESENCHYMAL TRANSITION IN CULTURES OF HUMAN ENDOTHELIAL CELLS}

doi:10.1136/annrheumdis-2013-203217.12

S Soldano, R Brizzolara, A Sulli, MA Cimmino, B Seriolo, P Montagna, M Cutolo. Research Laboratory and Academic Unit of Clinical Rheumatology, Department of Internal Medicine, University of Genova, Genova, Italy

Background and Objectives Endothelial/microvascular injury and myofibroblast activation are crucial events that seem to contribute to the development of fibrosis in connective tissue diseases such as systemic sclerosis (SSc) [1]. Endothelin-1 (ET-1) contributes to the fibrotic process by inducing myofibroblast activation and increased extracellular matrix (ECM) synthesis [2]. Recently, it has been shown that myofibroblast activation from altered microvasculature may arise from the transition of endothelial-to-mesenchymal cell process (EndoMT), thus expressing $\alpha$-smooth muscle actin $(\alpha$-SMA), vimentin and fibrillar collagens [3]. The study investigated the possible involvement of ET-1 in the EndoMT in cultures of human endothelial cells.

Material and Methods Human dermal microvascular endothelial cells (HMVEC, Lonza Clonetic, Switzerland) and human umbilical vein endothelial cells (HUVEC, Lonza), were treated with or without ET-1 (100 nM, Enzo Life Science, UK) for 3 and 6 days. The expression of $\alpha$-SMA, a marker of myofibroblast phenotype, and platelet endothelial cell adhesion molecule (PECAM-1 or CD31), a marker of endothelial phenotype, were evaluated by immunofluorescence (IF) and western blot analysis (WB) using primary antibodies to human $\alpha$-SMA (dilution 1:50 for IF and 1:100 for WB, Dako Cytomation, Denmark) and to human CD31 (dilution 1:200 for IF and 1:1000 for WB, CellSignalling Technology, Denver, USA), in accordance with recent evidences $[4,5]$. Data were obtained from four different experiments.
Results After 6 days of treatment ET-1 induced the expression of $\alpha$-SMA in cultures of HUVEC, which maintained their ability to express CD31. Interestingly, ET- 1 induced the $\alpha$-SMA expression also in cultures of HMVEC after 6 days of treatment, without modulating the expression of CD31 when compared to untreated cells and confirming the data obtained in HUVEC cultures. The results were obtained by IF and confirmed by WB.

Conclusions These preliminary results show that ET-1 seems to induce the $\alpha$-SMA expression in human endothelial cells thus supporting a possible direct involvement in promoting the EndoMT $[2,4-6]$. The implications in the fibrotic process that characterise SSc are under evaluation [7].

\section{References}

1. Wynn TA. J Pathol 2008; 214:199-210.

2. Batthacharyya S. et al, Nat Rev Rheumatol 2012; 8:42-54.

3. Piera-Velazquez S. et al, Am J Pathol 2011; 179:1074-80

4. Widyantoro B. et al, Circulation 2010; 8:2407-18.

5. Kitao A. et al, Am J Phatol 2009; 175:616-26.

6. Abraham D. et al, Arthrit Res Ther 2007; 9:doi.10.1186/ar2186.

7. Soldano S. et al, A\&R 2012; 64 (Supplement):S640

\section{A4.13 EVALUATION OF HISTOLOGY AND ADIPOKINE EXPRESSION OF SYNOVIAL TISSUE FROM PSORIATIC ARTHRITIS PATIENTS}

doi:10.1136/annrheumdis-2013-203217.13

Elisabeth Schmitt, Elena Neumann, Jörg Kriegsmann (Trier), Rainer Straub (Regensburg), U Müller-Ladner. Dept. Internal Medicine and Rheumatology, JustusLiebig-University Giessen, Kerckhoff-Klinik, Bad Nauheim, Germany

Background The pathophysiology of psoriatic arthritis (PsA) has still not been examined in minute detail as has rheumatoid arthritis (RA). As it is often difficult to differentiate between both diseases and there are numerous publications that synovial inflammation and cytokine pattern are different in PsA and RA, we analysed the synovial histological differences with respect to immunomodulatory adipokines resistin, visfatin and adiponectin.

Methods First, synovial tissues from affected joints with PsA were stained with hematoxylin/eosin to visualise the histology of the synovial membrane. Thereafter, macrophages were detected by immunohistochemistry using an anti-CD16 antibody, fibroblasts using an anti-vimentin antibody. Collagen type IV-antibodies were used to visualise vascular endothelial cells and anti-CD20 antibodies to identify B-lymphocytes. In addition, serial tissue sections were stained for resistin-, visfatin, and adiponectin.

Results In the majority of patients, the synovial tissue from PsA showed no hyperplasia of the lining layer in comparison to RA (lining thickness in \% of PsA patients: 1 cell layer, 64\%; 2-3 cell layers, $21 \%$; $>4$ cell layers, $24 \%$. Vice versa, an increased cellularity in the sublining could be detected. In PsA, a lower number of immune-cell follicles were observed although the number of synovial vessels was increased. In all samples, cellular adipokine signals were detectable. Adiponectin was mainly located in vessels showing a strong expression of this molecule. In contrast to $\mathrm{RA}$, the expression of adiponectin in the lining layer was completely absent in most PsA patients. Resistin was detectable in the sublining but also in the lining layer. A strong expression of visfatin could be found in the sublining, and to a lower extent in the lining layer. There was no association between the localisation of the adipokines and the quantity or of compact inflammatory infiltrates.

Conclusions The synovial membranes from PsA show clear differences to those of RA-affected joints. Adipokine expression is -in part- also different between RA and PsA patients. Here, specifically adiponectin is more prominent in the RA than in the PsA lining layer. Adipokines may therefore play different roles in inflammatory processes operative in these chronic rheumatic diseases. 